\title{
Modeling of the roller pressing of fibrous materials
}

\author{
Shavkat Khurramov * , Gayrat Bakhadirov, Eshmurod Buriev, and Dildora Abduxalikova \\ Tashkent Institute of Architecture and Civil Engineering, Tashkent, Uzbekistan
}

\begin{abstract}
The work is devoted to modeling the regularities of hydraulic pressure distribution and fluid removal from the fibrous material in the contact zone. It was found that the hydraulic pressure in the compression zone increases from zero at the initial point of contact to a maximum at the point of maximum compression of the fibrous material. The patterns of distribution of hydraulic pressure in the recovery zone depend on the length of the section, where the fluid moves from the layer of material to the elastic coatings. It was revealed that the amount of liquid removed at the beginning of the compression zone grows faster, then the growth rate is much lower, and at the end of the compression zone, the removed liquid is stabilized. The patterns of change in the removed fluid in the recovery zone depend on the angle, which determines the position of the point where the fluid changes direction.
\end{abstract}

\section{Introduction}

A special group in the technology of mechanical processing of materials in roller machines is the roller pressing of fibrous materials.

The roller pressing of fibrous material consists of two aspects - contact interaction and hydraulic interaction. In this case, a change in the indices of the first aspect affects the change in the indices of the second aspect and vice versa. Therefore, the study of one phenomenon without considering the second phenomenon does not allow obtaining reliable parameters of the technological process.

Following the two phenomena, the theory of roller pressing of fibrous materials presents a joint solution of two problems - contact and hydraulic problems.

Contact problems of the theory of roller pressing of fibrous materials have been sufficiently fully solved in works [1-8].

\footnotetext{
*Corresponding author: shavkat-xurramov59@mail.ru
} 


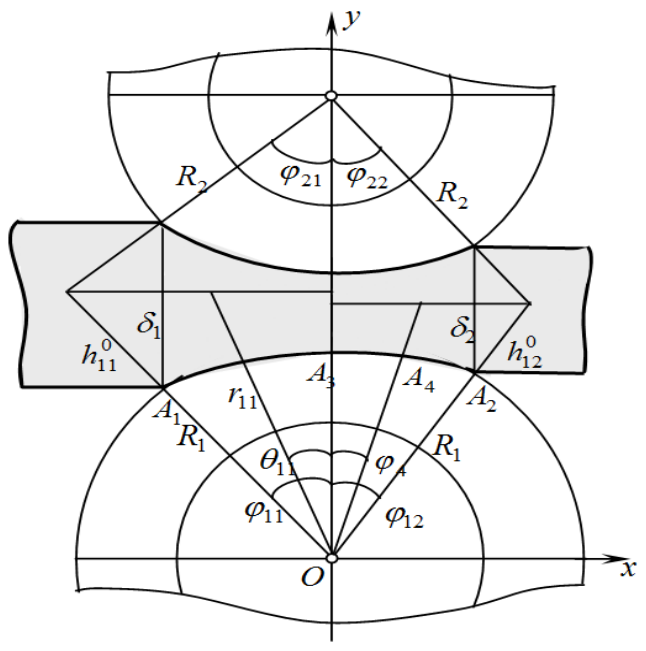

Fig. 1. Scheme of roller squeezing machine

Analysis of works devoted to the main hydraulic problems, such as modeling the regularities of the distribution of hydraulic pressure [9-16] and modeling the residual moisture content of fibrous material [17-23], showed that the existing models of the distribution of hydraulic pressure and changes in residual moisture were obtained with the introduction of two-roll models and materials that do not correspond to the real physical phenomena of the roller pressing of fibrous materials. In addition, the solutions of hydraulic problems in them are not related to the contact problems of two-roll squeezing modules.

The work is devoted to solving the problem of modeling the regularities of the distribution of hydraulic pressure and changes in the residual moisture content of the squeezed material, taking into account the results of solving the contact problems of tworoll squeezing modules.

\section{Materials and Methods}

Consider a two-roll squeezing module (Figure 1), in which the rollers have unequal diameters and elastic coatings made of materials of different stiffness and friction coefficients; both rollers are driven, the layer of material has a uniform thickness.

The lower roll contact curve (curve $A_{1} A_{2}$ ) consists of two zones $A_{1} A_{3}$ and $A_{3} A_{2}$. The compression of material and the roller covering occurs in the zone $A_{1} A_{3}$, and the recovery occurs in the zone $A_{3} A_{2}$.

First, consider the process of fluid filtration in the compression zone $A_{1} A_{3}$. In the compression zone, the liquid moves from the material to the roller coatings along the polar radius [5].

To solve the problems of the theory of roller pressing, it is necessary to develop the models of the roll contact curves and filtration rates in the area of pressing.

The model of the contact curve of the lower roller in the compression zone for the considered two-roll module has the form [2].

$$
r_{11}=\frac{R_{1}}{1+k_{11} \lambda_{11}}\left(1+k_{11} \lambda_{11} \frac{\cos \phi_{11}}{\cos \theta_{11}}\right)
$$


where $k_{11}=\frac{n_{11}}{m_{1}}, \lambda_{11}=\frac{d r_{11}}{d h_{1}}-$ are the ratio of strain-hardening coefficients and strain velocities of the roll coating and the layer of material under compression, respectively.

Hence

$$
r_{11}^{\prime}=\frac{k_{11} \lambda_{11} R_{1}}{1+k_{11} \lambda_{11}} \cos \varphi_{11} \frac{\sin \theta_{11}}{\cos ^{2} \theta_{11}}
$$

In [16], models of the filtration rates of the liquid flowing through the contact curve of the lower roller were determined

$$
\begin{aligned}
& u_{11 r}=b_{11}\left(\theta_{11}^{3}+\varphi_{11}^{3}\right)-\varphi_{11} \leq \theta_{11} \leq 0 \\
& u_{12 r}=b_{12}\left(\varphi_{14}^{3}-\theta_{12}^{3}\right), \quad 0 \leq \theta_{12} \leq \varphi_{12}
\end{aligned}
$$

where $\varphi_{14}=\varsigma_{1} \varphi_{12}, 0<\varsigma_{1} \leq 1$.

In [13], assuming the working hypothesis of the orthogonality of the maximum and minimum porosity, the applicability of the generalized Darcy's law to an anisotropic medium was established

$$
\frac{\partial P_{r}}{\partial r}=-\gamma \frac{u_{r}}{k_{\theta}}
$$

and found the formula for the filtration coefficient depending on the direction

$$
\frac{1}{K_{\theta}}=\frac{\cos ^{2} \theta}{K_{\max }}+\frac{\sin ^{2} \theta}{K_{\min }}
$$

where $P_{r}, u_{r}$-are the hydraulic pressure and filtration rate in direction $r ; K_{\max }-$ is the filtration

coefficient (maximum) along the axis $O y ; K_{\min }-$ is the filtration coefficient (minimum) along the

axis $O x ; \theta-$ is the angle that defines the direction $r ; \gamma-$ is the fluid viscosity coefficient.

According to formulas (3), (5) and (6), we obtain

$$
\frac{\partial P_{11 r}}{\partial r_{11}}=-\gamma b_{11}\left(\theta_{11}^{3}+\varphi_{11}^{3}\right)\left(\frac{\cos ^{2} \theta_{11}}{K_{11 \max }}+\frac{\sin ^{2} \theta_{11}}{K_{11 \text { min }}}\right)
$$

or making assumptions that $\cos ^{2} \theta_{11}^{2} \approx 1-\theta_{11}^{2}, \sin ^{2} \theta_{11} \approx \theta_{11}^{2}$

$$
d P_{11}=-\gamma b_{11}\left(\theta_{11}^{3}+\varphi_{11}^{3}\right)\left(\frac{1-\theta_{11}^{2}}{K_{11 \max }}+\frac{\theta_{11}^{2}}{K_{11 \min }}\right) \frac{d r_{11}}{d \theta_{11}} d \theta_{11}
$$


We substitute expression $\frac{d r_{11}}{d \theta_{11}}$ from equation (2) into this formula

$$
d P_{11}=-\frac{\mathcal{W}_{m} R_{1}^{2} k_{11} \lambda_{11} \cos ^{2} \varphi_{11}}{3 h_{11}^{0}\left(1+k_{11} \lambda_{11}\right)^{2}\left(1+k_{11} \lambda_{11} \cos \varphi_{11}\right)}\left(\frac{1-\theta_{11}^{2}}{K_{11 \max }}+\frac{\theta_{11}^{2}}{K_{11 \mathrm{~min}}}\right) \frac{\left(\varphi_{11}^{3}+\theta_{11}^{3}\right) \theta_{11}}{1-\theta_{11}^{2}} d \theta_{11}
$$

After integration, we obtain

$$
\begin{gathered}
P_{11}=-\frac{\mathcal{W}_{m} R_{1}^{2} k_{11} \lambda_{11} \cos ^{2} \varphi_{11}}{3 h_{11}^{0}\left(1+k_{11} \lambda_{11}\right)^{2}\left(1+k_{11} \lambda_{11} \cos \varphi_{11}\right)}\left(\frac{1}{K_{11 \max }}\left(\frac{1}{2} \varphi_{11}^{3} \theta_{11}^{2}+\frac{1}{5} \theta_{11}^{5}\right)-\right. \\
\left.-\frac{1}{k_{11 \text { min }}}\left(\varphi_{11}^{3}\left(\frac{1}{2} \theta_{11}^{2}+\frac{1}{2} \ln \left|1-\theta_{11}^{2}\right|\right)+\frac{1}{5} \theta_{11}^{5}+\frac{1}{3} \theta_{11}^{3}+\theta_{11}-\frac{1}{2} \ln \left|\frac{1+\theta_{11}}{1-\theta_{12}}\right|\right)\right)+C_{11} .
\end{gathered}
$$

Expanding the logarithmic functions in a series and limiting ourselves to terms up to the fifth power relative $\theta_{11}$, we obtain

$$
P_{11}=-c_{11}\left(5 \varphi_{11}^{3} \theta_{11}^{2}+2 \theta_{11}^{5}\right)+C_{11}
$$

where $c_{11}=\frac{\mathcal{N}_{m} R_{1}^{2} k_{11} \lambda_{11} \cos ^{2} \varphi_{11}}{30 K_{11 \max } h_{11}^{0}\left(1+k_{11} \lambda_{11}\right)^{2}\left(1+k_{11} \lambda_{11} \cos \varphi_{11}\right)}$.

The integration constant $C_{11}$ is determined by the initial condition $P_{11}\left(-\varphi_{11}\right)=0$ :

$$
P_{11}=c_{11}\left(3 \varphi_{11}^{5}-5 \varphi_{11}^{3} \theta_{11}^{2}-2 \theta_{11}^{5}\right), \quad-\varphi_{11} \leq \theta_{11} \leq 0
$$

In the recovery zone, the liquid, to the left of the certain point $A_{4}$, moves from the fibrous material to the roller covering, and to the right of this point, it moves to the fibrous material [13]. Point $A_{4}$ defined by angle $\varphi_{14}=\varsigma_{1} \varphi_{12}$, where $0<\zeta_{1} \leq 1$, divides the recovery zones into two sections: the first section where $0 \leq \theta_{12} \leq \varphi_{14}$ and the second section where, $\varphi_{14} \leq \theta_{12} \leq \varphi_{12}$.

The distribution of hydraulic pressure along the contact curve of the lower roller in the first section of the recovery zone is determined similar to (10):

$$
P_{12}=c_{12}\left(3 \varphi_{14}^{5}-5 \varphi_{14}^{3} \theta_{12}^{2}+2 \theta_{12}^{5}\right), \quad 0 \leq \theta_{12} \leq \varphi_{14},
$$

where $c_{12}=\frac{\mathcal{w}_{m} R_{1}^{2} k_{12} \lambda_{12} \cos ^{2} \varphi_{12}}{30 K_{12 \max } h_{12}^{0}\left(1+k_{12} \lambda_{12}\right)^{2}\left(1+k_{12} \lambda_{12} \cos \varphi_{12}\right)}$.

At the points of the second section, it is $u_{12 x}\left(\theta_{12}\right)=0$. Then it follows from equation (4), (5) and (6) that 


$$
d P_{12}=-\frac{\not b_{12}}{K_{11 \max }}\left(\varphi_{14}^{3}-\theta_{14}^{3}\right) \frac{d y_{12}}{d \theta_{12}} d \theta_{12}
$$

In the second section of the recovery zone $A_{4} A_{2}$, the equation $y_{12}=r_{12} \cos \theta_{12}$ holds.

Using expressions similar to (1) and (2) and making assumptions that $\sin \theta_{11} \approx \theta_{11}$, we obtain

$$
\frac{d y_{12}}{d \theta_{12}}=r_{12}^{\prime} \cos \theta_{12}-r_{12} \sin \theta_{12}=-\frac{R_{1}}{1+k_{12} \lambda_{12}} \theta_{12}
$$

Taking into account equation (13), from (12), we obtain

$$
d P_{12}=\frac{R_{1}^{2} \gamma v_{m} \cos \varphi_{12}}{3 K_{11 \max } h_{12}^{0}\left(1+k_{12} \lambda_{12}\right)\left(1+k_{12} \lambda_{12} \cos \varphi_{12}\right.}\left(\varphi_{14}^{3}-\theta_{14}^{3}\right) \theta_{12} d \theta_{12}
$$

Integrating (14) and using the boundary condition $P_{12}\left(\varphi_{14}\right)=0$, we obtain

$$
P_{12}=c_{13}\left(5 \varphi_{14}^{3} \theta_{12}^{2}-2 \theta_{12}^{5}-3 \varphi_{14}^{5}\right), \quad \varphi_{14} \leq \theta_{12} \leq \varphi_{12},
$$

where $\quad c_{13}=\frac{\mathcal{N}_{m} R_{1}^{2} \cos \varphi_{12}}{30 K_{12 \max } h_{12}^{0}\left(1+k_{12} \lambda_{12}\right)^{2}\left(1+k_{12} \lambda_{12} \cos \varphi_{12}\right)}$.

Thus, the distribution of hydraulic pressure along the contact curve of the lower roller is described by formulas (10), (11) and (15).

The distribution of hydraulic pressure along the contact curve of the upper roller is determined similarly.

$$
P_{21}=c_{21}\left(3 \varphi_{21}^{5}-5 \varphi_{21}^{3} \theta_{21}^{2}-2 \theta_{21}^{5}\right),-\varphi_{21} \leq \theta_{21} \leq 0
$$

where $\quad c_{21}=\frac{\mathcal{N}_{m} R_{2}^{2} k_{21} \lambda_{21} \cos ^{2} \varphi_{21}}{30 K_{21 \max } h_{21}^{0}\left(1+k_{21} \lambda_{21}\right)^{2}\left(1+k_{21} \lambda_{21} \cos \varphi_{21}\right)}$;

$$
P_{22}=c_{22}\left(3 \varphi_{24}^{5}-5 \varphi_{24}^{3} \theta_{22}^{2}+2 \theta_{22}^{5}\right), \quad 0 \leq \theta_{22} \leq \varphi_{24},
$$

where $\quad c_{22}=\frac{\mathcal{N}_{m} R_{2}^{2} k_{22} \lambda_{22} \cos ^{2} \varphi_{22}}{30 K_{22 \max } h_{22}^{0}\left(1+k_{22} \lambda_{22}\right)^{2}\left(1+k_{22} \lambda_{22} \cos \varphi_{22}\right)}$;

$$
P_{22}=c_{23}\left(5 \varphi_{24}^{3} \theta_{22}^{2}-2 \theta_{22}^{5}-3 \varphi_{24}^{5}\right), \quad \varphi_{24} \leq \theta_{22} \leq \varphi_{22},
$$


Where $c_{23}=\frac{\psi_{m} R_{2}^{2} \cos \varphi_{22}}{30 K_{22 \max } h_{22}^{0}\left(1+k_{22} \lambda_{22}\right)^{2}\left(1+k_{22} \lambda_{22} \cos \varphi_{22}\right)}, \varphi_{24}=\varsigma_{2} \varphi_{22}, 0<\varsigma_{2} \leq 1$.

It is known [13] that the amount of extracted liquid flowing along the contact surface of the roller can be determined by

$$
d Q=B \rho u_{r} d h
$$

where $B-$ is the width of the fibrous material layer; $\rho$-is the fluid density.

In the compression zone $A_{1} A_{3}$, the strain of the layer of material is expressed by the following equation

$$
h_{11}=r_{11} \cos \theta_{11}-R_{1} \cos \varphi_{11}
$$

Taking into account expressions (1), (2) and the assumption $\sin \theta_{11} \approx \theta_{11}$, we determine

$$
d Q_{11}=-\frac{\rho B R_{1} b_{11}}{1+k_{11} \lambda_{11}}\left(\theta_{11}^{3}+\varphi_{11}^{3}\right) \theta_{11} d \theta_{11}
$$

Integrating (20) and using the boundary condition $Q_{11}\left(-\varphi_{11}\right)=0$, we obtain

$$
Q_{11}=\alpha_{11}\left(3 \varphi_{11}^{5}-2 \theta_{11}^{5}-5 \varphi_{11}^{3} \theta_{11}^{2}\right),-\varphi_{11} \leq \theta_{11} \leq 0,
$$

where $\alpha_{11}=\frac{\rho v_{m} B R_{1}^{2} \cos \varphi_{11}}{30 h_{11}^{0}\left(1+k_{11} \lambda_{11}\right)^{2}\left(1+k_{11} \lambda_{11} \cos \varphi_{11}\right)}$.

The amount of extracted liquid flowing through the surface of the compression zone is determined by its flow rate at point $A_{3}$, that is, by moisture content $Q_{11}(0)$ :

$$
Q_{11}(0)=3 \alpha_{11} \varphi_{11}^{5}
$$

In the first section of the recovery zone, the strain of the layer of material has the form

$$
h_{12}=r_{12}(0)-r_{12} \cos \theta_{12} \text {. }
$$

Similar to (20), we obtain

$$
d Q_{12}=\frac{\rho B R_{1} b_{12}}{1+k_{12} \lambda_{12}}\left(\varphi_{14}^{3}-\theta_{12}^{3}\right) \theta_{12} d \theta_{12}
$$

after integrating and using the condition $Q_{12}(0)=Q_{11}(0)=3 \alpha_{11} \varphi_{11}^{5}$, we obtain

$$
Q_{12}=3 \alpha_{11} \varphi_{11}^{5}+\alpha_{12}\left(5 \varphi_{14}^{3} \theta_{12}^{2}-2 \theta_{12}^{5}\right), 0 \leq \theta_{12} \leq \varphi_{14},
$$


where $\alpha_{12}=\frac{v_{m} \rho R_{1}^{2} \cos \varphi_{12}}{30 h_{12}^{0}\left(1+k_{12} \lambda_{12}\right)^{2}\left(1+k_{12} \lambda_{12} \cos \varphi_{12}\right)}$.

The amount of pressed liquid flowed from the layer of material through the surfaces of the compression zone, and the first section of the recovery zone is determined by the moisture content $Q_{12}\left(\varphi_{14}\right)$ :

$$
Q_{12}\left(\varphi_{14}\right)=3 \alpha_{11} \varphi_{11}^{5}+3 \alpha_{12} \varphi_{14}^{5}
$$

In the second section of the recovery zone, the strain of the fibrous material is

$$
h_{12}=r_{12}\left(\varphi_{14}\right)-r_{12} \cos \theta_{12} \text {. }
$$

Similar to (20), we obtain

$$
d Q_{12}=\frac{\rho B R_{1} b_{12}}{1+k_{12} \lambda_{12}}\left(\varphi_{14}^{3}-\theta_{12}^{3}\right) \theta_{12} d \theta_{12}
$$

or, after integrating and using condition (25), we obtain

$$
Q_{12}=3 \alpha_{11} \varphi_{11}^{5}+\alpha_{12}\left(5 \varphi_{14}^{3} \theta_{12}^{2}-2 \theta_{12}^{5}\right), \quad \varphi_{14} \leq \theta_{12} \leq \varphi_{12}
$$

Generalizing formulas (34) and (26), we obtain

$$
Q_{12}=3 \alpha_{11} \varphi_{11}^{5}+\alpha_{12}\left(5 \varphi_{14}^{3} \theta_{12}^{2}-2 \theta_{12}^{5}\right), \varphi_{14} \leq \theta_{12} \leq \varphi_{12}
$$

where $\varphi_{14}=\varsigma_{1} \varphi_{12}, 0<\varsigma_{1} \leq 1$.

The amount of liquid extracted from the material through the contact surfaces of the lower roller is determined by its flow rate at point $A_{2}$, that is, by moisture content $Q_{12}\left(\varphi_{12}\right)$ :

$$
Q_{12}\left(\varphi_{12}\right)=3 \alpha_{11} \varphi_{11}^{5}+\alpha_{12}\left(5 \varphi_{14}^{3} \varphi_{12}^{2}-2 \varphi_{12}^{5}\right)
$$

The amount of liquid that flowed through the contact surfaces of the upper roller is determined similarly:

$$
Q_{22}\left(\varphi_{22}\right)=3 \alpha_{21} \varphi_{21}^{5}+\alpha_{22}\left(5 \varphi_{24}^{3} \varphi_{22}^{2}-2 \varphi_{22}^{5}\right)
$$

The amount of liquid extracted from the layer of material during the pressing process is equal to the sum of the number of liquids extracted through the contact surfaces of the lower and upper rollers:

$$
Q_{y}=3 \alpha_{11} \varphi_{11}^{5}+\alpha_{12}\left(5 \varphi_{14}^{3} \varphi_{12}^{2}-2 \varphi_{12}^{5}\right)+3 \alpha_{21} \varphi_{21}^{5}+\alpha_{22}\left(5 \varphi_{24}^{3} \varphi_{22}^{2}-2 \varphi_{22}^{5}\right)
$$


Knowing the amount of extracted liquid, the moisture squeezed out from the processed material under pressing is determined by the expression given in:

$$
W_{\mathrm{y}}=\frac{Q_{\mathrm{y}}}{\rho B v_{m}} 100 \%
$$

where $v_{m}-$ is the velocity of the material.

In the case of roller pressing, the following equation holds:

$$
W_{o}=W_{n}-W_{y}
$$

where $W_{o}, W_{n}-$ are the residual and initial moisture contents of the processed material, respectively.

Thus, the residual moisture of the roller pressing of the fibrous material is determined by the following expressions:

$$
W_{0}=W_{\mathrm{H}}-\frac{1}{\rho B v_{m}}\left(3 \alpha_{11} \phi_{11}^{5}+\alpha_{12}\left(5 \phi_{14}^{3} \phi_{12}^{2}-2 \phi_{12}^{5}\right)+3 \alpha_{21} \phi_{21}^{5}+\alpha_{22}\left(5 \phi_{24}^{3} \phi_{22}^{2}-2 \phi_{22}^{5}\right)\right)
$$

\section{Results and Discussion}

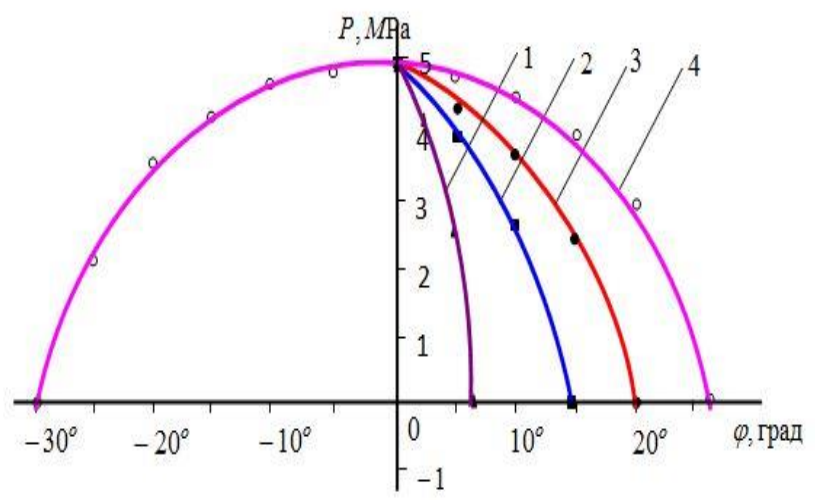

Fig. 2. Graphs of changes in the hydraulic pressure in the contact area:

$$
1-\varphi_{14}=0.25 \varphi_{12} ; 2-\varphi_{14}=0.5 \varphi_{12} ; 3-\varphi_{14}=0.75 \varphi_{12}, 4-\varphi_{14}=\varphi_{12} \text {. }
$$

On the graphs of the distribution of hydraulic pressures in the contact zone of the rolls, shown in Figure 2, it follows that the hydraulic pressure in the compression zone increases from zero at the initial point of contact to a maximum at the point of maximum compression of the material. The distribution of hydraulic pressure in the contact zone depends on the length of its part, where the liquid passes from the material layer to the roll coating. 




Fig.3. Graphs of changes in the removed liquid in the contact zone

$$
1-\varphi_{14}=0.25 \varphi_{12} ; 2-\varphi_{14}=0.5 \varphi_{12} ; 3-\varphi_{14}=0.75 \varphi_{12}, 4-\varphi_{14}=\varphi_{12} \text {. }
$$

The patterns of change in the extracted fluid in the recovery zone depend on the value of $\zeta_{i}=\sqrt[3]{0.4}=0.74$, which determines the position of the point where the fluid changes its direction. At $0<\zeta_{i}<0.74$, the amount of squeezed moisture after pressing is less than at the end of the compression zone. In this case, a layer of material from the roller covering reabsorbs moisture. At $0.74 \leq \zeta_{i}<1$, the amount of squeezed moisture after pressing is greater than at the end of the compression zone. At the same time, at the beginning of the recovery zone, the liquid moves from the coating into the layer of material, and at the end of the recovery zone, the reabsorption of moisture from the roller covering occurs, and the amount of liquid extracted from the layer of material in the recovery zone is greater than the amount absorbed. At a critical value of $\zeta_{i}=0.74$, the amount of moisture extracted under pressing is equal to the amount of moisture extracted at the end of the compression zone; that is, the amount of extracted and absorbed liquid in the recovery zone is equal.

The residual moisture content of the fibrous material depends on the value of $\zeta_{i}$. At $0<\zeta_{i}<0.74$, the residual moisture is greater than the moisture content at the end of the compression zone, which is associated with the reverse movement of liquid from the coating into the layer of material. At $0.74 \leq \zeta_{i} \leq 1$, the residual moisture is less than the moisture content at the end of the compression zone due to the liquid extraction in the recovery zone. At $\zeta_{i}=1$, the residual moisture content has the lowest value.

\section{Conclusions}

1. Mathematical models of the patterns of distribution of hydraulic pressure and residual moisture of the squeezed material were obtained.

2. The hydraulic pressure in the compression zone increases from zero at the initial point of contact to a maximum at the point of maximum compression of the fibrous material. The patterns of distribution of hydraulic pressure in the recovery zone depend on the length of the section, where the liquid moves from the layer of material to the roller coatings.

3. The amount of liquid extracted at the beginning of the compression zone increases faster, which is associated with a rapid increase in the compression of the layer of material. Then the rate of growth of the extracted fluid becomes slower, and at the end of the compression zone, the amount of extracted fluid stabilizes due to a decrease in the compression strain of the layer of material. 


\section{References}

1. Alexa V, Ratiu S and Kiss I. IOP Conference Series: Materials Science and Engineering. 106, (2019).

2. Khurramov Sh R, IOP Conf. Series: Earth and Environmental Science. 614, 012096 1205. (2020)

3. Xiawei F, Xiochen W, Quan Y, Juquan S, Advances in Mechanical Engineering. pp 811. (2019).

4. Khurramov, Sh.R. Journal of Physics: Conf. Series.1546, 012132. (2020)

5. Pan S C, Huang M N, Tzou G Y, Syu S W, Journal of Materials Processing Technology 177, pp 114-120. (2019)

6. Khurramov Sh R, Abdukarimov A, Khalturaev F S., Kurbanova F Z. IOP Conference Series: Materials Science and Engineering, p. 916, 012051, (2019)

7. Khurramov Sh R, Kurbanova F Z. IOP Conf. Series: Earth and Environmental Science. 614 012098. (2020)

8. Khurramov Sh R, Khalturayev F S. IOP Conf. Series: Earth and Environmental Science. 614, 012097, (2020),

9. Kataya M, Kirmanen J and Timonen J, Nordic Pulp and Paper Research Journal 10 (3) pp 162-166. (1995).

10. Iliev O, Printsypar G and Rief S, J Transport in Porous Media 92 pp 41-59 10 162166. (2012).

11. El-Hosseiny F, Nordic Pulp and Paper Research Journal 6 pp 30-34, (1991).

12. Bezanovic D, Duijn C J and Kaasschieter, J Transport in Porous Media 67, pp 171187. (2007).

13. Novikov NE, Pressing of paper web Moscow, Forest industry.(1972)

14. Iliev O, Printsypar G and Rief, Journal of Engineering Mathematics 83 (1) pp 81-107. (2013).

15. Fitt A D, Howell P D, King J R, Please C P and Schwendeman D W, Europian Journal of ApplieMathematics 13 (3) pp 225-259. (2002).

16. Khurramov Sh.R. Conf. "Strength of structures, seismodynamics of buildings and structures" Uzbekistan, Tashkent pp 488-492. (2016).

17. Kataya M, Kirmanen J and Timonen J, J.Phys. D: Appl. Phys 25 pp 1053-1063 (1992)

18. McDonald D and Kerekes R J, J BioResources 12 pp 9520-9537. (2017)

19. Kerekes R J, McDonald E M and McDonald J D, Journal Science and Technology for Forest Products and Processes 3 pp 46-51 (2013).

20. McDonald D, Kerekes R J and Zhao J, J BioResources 15, pp 7319-7329. (2020)

21. Wahlstrom P B, Preprints of the 44th Appita Annual General Conf. Australia 211. (1990)

22. McDonald J D, Hamel J and Kerekes R J, Jjurnal of Pulp and Paper Science pp 401406. (2000)

23. Koivu V, Decain M, Geindreau C, Mattila K, Alaraudanjoki J, Bloch J F and Kataja M, In Proceedings in the $14^{\text {th }}$ Fundamental Research Symposium pp 437-454. (2009) 